\title{
Adaptive control of a 7-DOF exoskeleton robot with uncertainties on kinematics and dynamics
}

\author{
Brahim Brahmi a,*, Maarouf Saad ${ }^{\mathrm{a}}$, Jacqueline Tu Anh Thu Lam ${ }^{\mathrm{a}}$, Cristobal Ochoa Luna ${ }^{\mathrm{b}}$, \\ Philippe S. Archambault ${ }^{\mathrm{b}}$, Mohammad H. Rahman ${ }^{\mathrm{c}}$ \\ a Electrical Engineering Department, École de technologie supérieure, Montreal, Canada \\ ${ }^{\mathrm{b}}$ School of Physical E' Occupational Therapy, McGill University; Centre for Interdisciplinary Research in Rehabilitation of Greater Montreal. Montreal, Canada \\ ${ }^{c}$ Mechanical Engineering Department, University of Wisconsin-Milwaukee, WI, USA
}

\section{A R T I C L E I N F O}

\section{Article history:}

Received 7 June 2017

Revised 18 February 2018

Accepted 1 March 2018

Available online 9 March 2018

Recommended by X. Chen

\section{Keywords:}

Rehabilitation robots

Time delay control

Time delay error

Uncertainties, recursive control

\begin{abstract}
A B S T R A C T
In this paper, we propose a new adaptive visual tracking control approach based on sliding mode control in Cartesian space applied to an exoskeleton robot with uncertain kinematics and dynamics, taking into account uncertainties in visual system (camera) parameters. The adaptation of kinematic uncertainties is based on a filtered regressor kinematic matrix, whereas, the adaptation of dynamic uncertainties is based on a Time Delay Estimation approach. This is performed considering the Time Delay Error (TDR) to provide a control action capable of following the designed functional therapy tasks. A new recursive controller is combined with TDE in order to estimate the TDR and limit its effect. The proposed strategy does not need the accurate dynamic and kinematic models of the exoskeleton. The update laws are designed using Lyapunov theory to solve the adaptation problem methodically and to show the stability of the robot system. Experimental results confirm the effectiveness and feasibility of the designed approach.

(c) 2018 European Control Association. Published by Elsevier Ltd. All rights reserved.
\end{abstract}

\section{Introduction}

Recently, stroke and neurological diseases have become among the most important health-related problems in the world. Stroke survivors bear with disabilities following an accident that affects their quality of life [32,34]. Annually, worldwide, six million people die and five million live with persistent weakness, from the 15 million people suffering a stroke [37]. Physical therapy is the main treatment existing today. It is designed to relieve the patient from the impairment or/and injury and to improve his range of movement, functional capacity, and quality of life [14]. Thanks to robotics technology, modern rehabilitation treatment is supported by new devices named rehabilitation robots. This kind of robot is able to provide a wide range of physical therapy and overcome some of the limitations of conventional therapy. Many research teams participate in this field, among them, Assisted Rehabilitation and Measurement Guide (ARMin IV) [26]; Robotic Upper Extremity Repetitive Therapy (RUPERT) [3]; Saga University Exoskeleton For Upper Limb (SUEFUL-7) [20]. An important issue is that the de-

\footnotetext{
* Corresponding author.

E-mail addresses: brahim.brahmi.1@ens.etsmtl.ca (B. Brahmi),
} maarouf.saad@etsmtl.ca (M. Saad), cristobal.ochoa.luna@mail.mcgill.ca (C.O. Luna), philippe.archambault@mcgill.ca (P.S. Archambault), rahmanmh@uwm.edu (M.H. Rahman). sign of these robots must be harmonious with the human anatomy configuration. To provide a modern physical therapy for the upper limb, we have developed an exoskeleton robot named ETSMARSE. This robot is consistent with the human arm configuration and is capable of performing different rehabilitation movements $[6,4,9]$.

Generally, the dynamic parameters of an exoskeleton robot, for instance the ETS-MARSE, are hard to be modeled precisely because of the complexity of the mechanical design such as nonlinear friction forces, backlash, and the complexity of the actuators of the robot. In addition, the dynamic characteristics vary due to the different physiological conditions of the subjects, such as an external force caused by subject's muscular activity [6]. This kind of uncertain nonlinearities can be categorized as both parametric uncertainties and unknown nonlinear functions [29]. Additionally, in most applications using rehabilitation robots, the therapeutic tasks are expressed in Cartesian space. In this case, the nonlinear transformation functions or Jacobian matrix that allows the mapping from joint space to Cartesian space is assumed to be known, to ensure a perfect Cartesian control performance. However, when the feedback position of the robot's response is provided by a visual system, such as a camera or Kinect, the exoskeleton can be subject to uncertain kinematics. Due to firstly the uncertainties in camera parameters, secondly, when the human and exoskeleton carry/transfer an object with an unknown length and/or ori- 
entation [1]. In this case, it is difficult to derive the solution of inverse kinematics using this standard approach. Moreover, to realize human motion via an exoskeleton robot, it is essential to determine accurately the lengths of links, the joints, and the dimension of the object carried by the robot. Unfortunately, it is difficult to define these physical parameters exactly. Various solutions have been proposed to estimate these parameters [10,11,2,21,39]. However, almost none of these solutions consider the uncertainties of the camera parameters. This dilemma motivates us to set up a new control system capable of ensuring a sufficient performance in the presence of dynamic and kinematic uncertainties and unknown disturbances exist.

One of the research challenges on this class of robots is to develop a controller that can maneuver the human-exoskeleton system to mimic natural human upper extremity motion. It is remarkable from a natural human movement that the human does not need accurate information about kinematics and dynamics of the arm (or any object carried by upper extremity) to reach an object in space. Due to that, many control strategies have been designed to solve the problem of kinematic and dynamic uncertainties $[1,10,41,23,12,24]$. The main innovative point of these controllers is that the adaptation of the both kinematic/dynamic uncertainties has been provided, which makes the exoskeleton robot perform the human-like motion and supplies to the control system more flexibility to handle the uncertainties and parameters variation. However, the above controllers are based on the classical regressor matrix. These types of controllers assume that the robot is linear in a set of physical parameters and find a control law able to ensure the stability of this linear system only around its operating points [40]. In fact, the manipulator is highly nonlinear. So, the integration of this adaptation law may affect the stability of the system in the presence of even small disturbances [40]. Adaptive visual or image-based tracking control $[24,15,17,19,33,30]$ is one of the powerful approaches that has been developed to transact with the kinematic/dynamic uncertainties. This is due to their robustness practically to modeling and calibration errors [15]. However, these controllers are concentrated on uncertainties in nonlinear transformation functions or image Jacobian matrix but they ignored the uncertain kinematic/dynamic effects. Additionally, a few stability analyses are provided in the literature for visual tracking control with the uncertainties of kinematics/dynamics and in the presence of uncertainties in visual system (camera) parameters [13]. A Time Delay Estimation (TDE) approach may be considered to compensate the uncertainties $[42,16,36,22,18,43,7,8]$. The TDE has been implemented in many robotic systems with consistently good performance $[25,28]$. The TDE utilizes the previous response of the robot system, and the previous control input to provide new control actions able to provide an accurate approximation of uncertainty function. However, due to noisy measurements and nonlinearity of signals along the sampling time, a time delay error (TDR) exists, which would deteriorate the robustness and the accuracy of the robot. A through literature review revealed that no research work has proposed a systematic solution to eliminate the negative influence of this error.

\subsection{Main contribution}

All the papers that cited above, except TDE approach, are based on the conventional adaptive approaches (regressor function) and require a good knowledge of the robot system's parameters. Practically, it is impossible to define exactly the parameters of the robot system and the modelisation of the robot is typically uncertain. For these kinds of robots, the adaptation of the uncertainties function based on full dynamic is very complicated due to the high number of degrees of freedom (DOFs) of the robot. When the num- ber of DOFs of the robot increases, it is not straightforward to find the parameters of the robot. Usually, the parameter vector of the robot can be greater than 100 if its DOFs are greater only than four [5].

To address the above problems, we propose a new adaptive visual tracking control for an exoskeleton robot with high number of degrees of freedom (7-DOFs) based on extension of sliding mode, TDE approach and Jacobian transpose taking into consideration the Cartesian and joint spaces. This controller is designed to be robust and more flexible to deal with the kinematic and dynamic uncertainties taking into consideration the uncertainties in the visual system parameters, and to be more robust to the parameter variations. The contribution of this paper can be summarized in three points:

(i) Considering the unknown kinematics and dynamics with unknown external disturbances (different weight of the arm of each subject), adaptive visual controller incorporating with recursive control is developed to estimate the nonlinear kinematic and dynamic uncertainties with unknown disturbances and to drive the robot to follow the desired functional therapy activity and provide a smooth exoskeletonaided passive activity.

(ii) The unknown dynamics and external disturbances of the robot system can estimate easily using Time Delay Estimation (TDE) approach. This strategy employs only timedelayed knowledge about the previous control input of the system and its response state to provide an accurate estimation of uncertainties. The main feature of this method is that not influenced by the high degree of freedom of the robot and the size of the estimated parameters.

(iii) Using a new recursive control to reduce the effect of the Time Delay Error (TDR) and improve the robustness of the control system. Usually, this error limits the performance of TDE approach.

The proposed strategy is achieved based on the inner/outer loop structure of robotics system. This latter has some desirable characteristics such as the rapidity of the computation of the control system. In this case, the outer loop is designed to estimate the nonlinear kinematics parameters and uncertainties in the visual system (camera), and the Inner Loop is intended to provide a high-level of precision by compensating the unknown part of the dynamics using TDE approach and while considering the TDR. The recursive control here is designed to reduce the effect or TDR and improve the robustness of the TDE approach. The structure of the designed controller also aims to make the exoskeleton robot perform the human-like movement using the predefined trajectories of physical therapy tasks [14]. The stability of the Inner/Outer system and the convergence of its errors are formulated and demonstrated based on Lyapunov function. Compared with conventional approach $[10-12,30]$ that is applied on only 2DOFs planar robot, the designed strategy is characterized by the ease of implementation and high precision and robustness to the kinematic/dynamic uncertainties, unforeseen disturbances and uncertainties of camera parameters. The efficiency and the robustness of the proposed approach are validated with Cartesian trajectory tracking corresponding to passive physical therapy tasks [14].

The outline of the paper is organized as follows. The kinematics and dynamics of the robot are presented in the next section. The control scheme is described in section III. Experimental and comparison results are shown in section IV; finally, the conclusion is presented in section $\mathrm{V}$. 


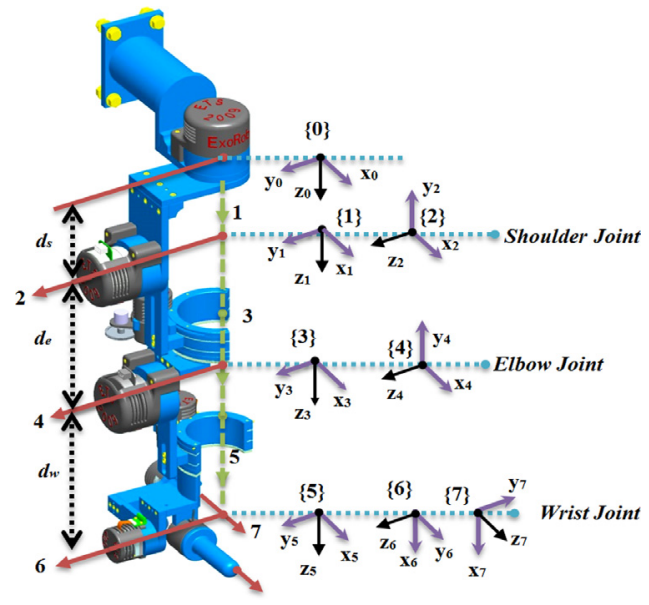

Fig. 1. Reference frames of ETS-MARSE.

\section{Characterization of ETS-MARSE robot: Kinematics and dynamics}

\subsection{Modeling of ETS-MARSE robot}

The ETS-MARSE is a redundant robot with 7DOFs, as shown in Fig. 1. It is designed to rehabilitate the impaired human upper limb. The design of the ETS-MARSE was originally inspired from the anatomy of the human arm. It was ergonomically designed to be comfortable for the subjects (robot users) during the rehabilitation sessions. The shoulder motion part (3DOF) is consisted of three joints: the first two joints are responsible for shoulder joint's vertical and horizontal extension/flexion motion, while the third joint is aimed to conduct the internal and external rotation of the shoulder joint. The elbow motion part (1 DOF) is responsible for elbow joint flexion/extension motion. The wrist motion support part of the ETS-MARSE is consisted of three joints: the first joint is designed to achieve pronation and supination movement of the forearm, the second joint and the third joint are designed to perform ulnar/radial deviation, and flexion/extension of the wrist respectively. The design of the ETS-MARSE has special features compared with the existing exoskeleton robots [35]. Among them, it has a comparatively low weight, an excellent power/weight ratio, can be easily fitted or removed, and is capable of adequately compensating for gravity. A new power carrying mechanism was included for supporting the shoulder joint internal/external rotation and for forearm pronation/supination. This exoskeleton robot can be used with a wide range of subjects, due to its adjustable link mechanism. All the key characteristics and contribution features of the ETS-MARSE and comparison with similar existing exoskeleton robots are summarized in [35].

\subsection{Dynamics of ETS-MARSE robot}

The dynamic behavior of ETS-MARSE manipulator is given by the following expression using the Lagrangian method [29]:

$M(\theta) \ddot{\theta}+C(\theta, \dot{\theta}) \dot{\theta}+F(\theta, \dot{\theta})+G(\theta)=\tau+\tau_{e x}$

where $\theta, \dot{\theta}$, and $\ddot{\theta} R^{7}$ are respectively the joints position, velocity, and acceleration vectors, $M(\theta) \in R^{7} \times 7$ is the symmetric and positive definite inertia matrix, $C(\theta, \dot{\theta}) \dot{\theta} \in R^{7}$ is the Coriolis and centrifugal vector, $G(\theta) \in R^{7}$ is the gravitational vector, $\tau \in R^{7}$ is the torque vector, $\tau_{e x} \in R^{7}$ is the external disturbances vector, and
$F(\theta, \dot{\theta}) \in R^{7}$ is the friction vector. Let us denote

$$
\left\{\begin{array}{l}
M(\theta)=M_{0}(\theta)+\Delta M(\theta) \\
C(\theta, \dot{\theta})=C_{0}(\theta, \dot{\theta})+\Delta C(\theta, \dot{\theta}) \\
G(\theta)=G_{0}(\theta)+\Delta G(\theta)
\end{array}\right.
$$

where $M_{0}(\theta), C_{0}(\theta, \dot{\theta})$, and $G_{0}(\theta)$ are respectively the known inertia matrix, Coriolis/centrifugal matrix, and gravity vector. $\Delta M(\theta)$, $\Delta C(\theta, \dot{\theta})$, and $\Delta G(\theta)$ are the uncertain parts.

Let us introduce a new variable such that: $\eta_{1}=\theta$ and $\eta_{2}=\dot{\theta}$; hence, the dynamic model expressed in (1) can be rewritten, without loss of generality, as follows:

$\left\{\begin{array}{l}\dot{\eta}_{1}=\eta_{2} \\ \dot{\eta}_{2}=U(t)+f(t)+H(t)\end{array}\right.$

with, $U(t)=U\left(\eta_{1}\right) ; H(t)=H\left(\eta_{1}, \eta_{2}, \dot{\eta}_{2}\right)$ and $f(t)=f\left(\eta_{1}, \eta_{2}\right)$. This notation is used in order to handle easily later with the control scheme. Where:

$$
\begin{aligned}
& \text { - } U(\mathrm{t})=M_{0}^{-1}(\theta) \tau \\
& \text { - } H(\mathrm{t})=M_{0}^{-1}(\theta)\left(\tau_{e x}-\Delta M(\theta) \ddot{\theta}-\Delta C(\theta, \dot{\theta}) \dot{\theta}-\Delta G(\theta)-F(\theta, \dot{\theta})\right) \text {, } \\
& \text { and } \\
& \text { - } f(\mathrm{t})=M_{0}^{-1}(\theta)\left(-C_{0}(\theta, \dot{\theta}) \dot{\theta}-G_{0}(\theta)\right)
\end{aligned}
$$

\subsection{Kinematics of ETS-MARSE robot}

In most applications of rehabilitation robots, the desired trajectory is expressed in Cartesian space [14]. The transformation from Cartesian space to joint space is done by a non-linear function named the Jacobian matrix. If the position $x$ of the end-effector of the robot is provided by a visual system such a camera or Kinect, the standard relation between Cartesian velocity and joint velocity is given as follows:

$\dot{x}=J\left(\eta_{1}\right) \eta_{2}$

where $J\left(\eta_{1}\right)=J_{c}(r) J_{R o}\left(\eta_{1}\right) \in R^{6 \times 7}$ is the total Jacobian matrix of the robot combined with the visual system. $J_{c}(r) \in R^{6 \times 6}$ is the image Jacobian matrix [12,24], $r \in R^{6}$ is the Cartesian position of the end-effector of the robot, $x \in R^{6}$ is the image feature parameters and $J_{R 0}\left(\eta_{1}\right) \in R^{6 \times 7}$ is the Jacobian matrix of the manipulator.

\subsection{Problem formulation}

One of the main objectives of research on this kind of robots is to design a controller able to make the human-exoskeleton system achieve movement without exact information of the kinematics and dynamics of the robotic system and to provide a smooth movement, conforming to physical therapy exercise while the designed control scheme ensures that the measured Cartesian positions $x$ of the robot tracks the desired Cartesian trajectory $x_{d}$. Before giving the control design methodology, we present the properties and the assumptions used in this paper.

Property 1. The known part of inertia matrix $M_{0}(\theta)$ is symmetric and positive definite for all $\theta \in R^{7}$ [29].

Property 2. Eq. (4) can be linear in a set of kinematics parameters like link lengths, which can be written as a linear combination of specified functions as given below [30]:

$\dot{x}=J\left(\eta_{1}, \gamma_{k}\right) \eta_{2}=Q\left(\eta_{1}, \eta_{2}\right) \gamma_{k}$

where $Q\left(\eta_{1}, \eta_{2}\right) \in R^{6 \times 6}$ is the kinematic regressor matrix and $\gamma_{k} \in$ $R^{6}$ is the kinematics parameters vector.

Assumption 1. The joint position and joint velocity are measured.

Assumption 2. All kinematic singularities are avoided. 
Assumption 3. Since the dynamic model of the robot is continuous and bounded, we assume that the function $H(t)$ is locally Lipschitz function $[38,27]$.

Assumption 4. The desired trajectory is bounded.

Assumption 5. The external disturbance $\tau_{e x}$ is supposed to be continuous, has finite energy, and satisfies $\left\|\tau_{e x}\right\| \leq \vartheta$, with an unknown positive disturbance boundary $\vartheta$.

\section{Control design}

The control of a robotic system always needs a precise input measurement to provide a good performance of this system. Particularly, in the case when we have a position feedback from visual devices, like a camera or Kinect. Since no sensor is available to measure the Cartesian velocity input, a linear filter can be used to obtain this variable such that:

$\left(\frac{d}{d t}+\Lambda\right) z=\Lambda \dot{x}$

The signal $z \in R^{6}$ is obtained from the measured position $x \in R^{6}$. $\Lambda$ is a positive constant. Substituting (5) into (6), we can rewrite (6) such that

$\left(\frac{d}{d t}+\Lambda\right) z=\Lambda Q\left(\eta_{1}, \eta_{2}\right) \gamma_{k}$

where:

$z=\left(\frac{\Lambda}{\frac{d}{d t}+\Lambda}\right) Q\left(\eta_{1}, \eta_{2}\right) \gamma_{k}=\phi(t) \gamma_{k}$

where $\phi(t) \in R^{6 \times 6}$ is a filtered function of $Q\left(\eta_{1}, \eta_{2}\right)$. Usually the robot begins from the rest position, hence: $z(0)=0$ and $\phi(0)=0$ [12].

We can now determine the Cartesian position error, Cartesian velocity error, and estimated Cartesian velocity error as follows:

$\left\{\begin{array}{l}e_{x}=x-x_{d} \\ \dot{e}_{x}=\dot{x}-\dot{x}_{d} \\ \dot{\hat{e}}_{x}=\dot{\hat{x}}-\dot{x}_{d}\end{array}\right.$

where, $x_{d} \in R^{6}$ is the desired Cartesian trajectory, $x \in R^{6}$ is the measured position and $\dot{\hat{x}} \in R^{6}$ is the estimated measured velocity.

Now, we define the vector of required Cartesian velocity as:

$\dot{x}_{r}=\dot{x}_{d}-\beta e_{x}$

where $\dot{x}_{r} \in R^{6}$ and $\beta$ is a positive constant. Differentiating (10) with respect to time yields:

$\ddot{x}_{r}=\ddot{x}_{d}-\beta \dot{e}_{x}$

Considering an uncertain kinematics, the Jacobian matrix is uncertain. In this case (5) is rewritten as follows:

$\dot{\hat{x}}=\hat{J}\left(\eta_{1}, \hat{\gamma}_{k}\right) \eta_{2}=Q\left(\eta_{1}, \eta_{2}\right) \hat{\gamma}_{k}$

Using (12), we define the estimated sliding Cartesian surface vector as follows:

$\hat{s}_{x}=\dot{\hat{x}}-\dot{x}_{r}=\hat{J}\left(\eta_{1}, \hat{\gamma}_{k}\right) \eta_{2}-\dot{x}_{r}$

Differentiating (13) with respect to time yields:

$\dot{\hat{\hat{s}}}=\ddot{\hat{x}}-\ddot{x}_{r}=\hat{J}\left(\eta_{1}, \hat{\gamma}_{k}\right) \dot{\eta}_{2}+\dot{\hat{J}}\left(\eta_{1}, \hat{\gamma}_{k}\right) \eta_{2}-\ddot{x}_{r}$

Now, we can define the required joint velocity vector as:

$\dot{\eta}_{r}=\hat{J}^{+}\left(\eta_{1}, \hat{\gamma}_{k}\right) \dot{x}_{r}$

where $\hat{J}^{+}\left(\eta_{1}, \hat{\gamma}_{k}\right)=\hat{J}^{T}\left(\eta_{1}, \hat{\gamma}_{k}\right)\left(\hat{J}\left(\eta_{1}, \hat{\gamma}_{k}\right) \hat{J}^{T}\left(\eta_{1}, \hat{\gamma}_{k}\right)\right)^{-1}$ is the pseudoJacobian matrix.
Differentiating (15) with respect to time:

$\ddot{\eta}_{r}=\hat{J}^{+}\left(\eta_{1}, \hat{\gamma}_{k}\right) \ddot{x}_{r}+\dot{\hat{J}}^{+}\left(\eta_{1}, \hat{\gamma}_{k}\right) \dot{x}_{r}$

It is important also to define the sliding joint surface vector. By using (10), (12) and (15) we have:

$s=\eta_{2}-\dot{\eta}_{r}=\hat{J}^{+}\left(\eta_{1}, \hat{\gamma}_{k}\right)\left[\left(\dot{\hat{x}}-\dot{x}_{d}\right)+\beta e_{x}\right]=\hat{J}^{+}\left(\eta_{1}, \hat{\gamma}_{k}\right) \hat{s}_{x}$

The time derivative of relation (17) gives

$\dot{s}=\dot{\eta}_{2}-\ddot{\eta}_{r}=\hat{J}^{+}\left(\eta_{1}, \hat{\gamma}_{k}\right) \dot{\hat{s}}_{x}+\dot{\hat{J}}^{+}\left(\eta_{1}, \hat{\gamma}_{k}\right) \hat{s}_{x}$

Substituting (18) into (3), the exoskeleton robot system (3) can be written as follows:

$\left\{\begin{array}{l}s=\dot{\eta}_{1}-\dot{\eta}_{r} \\ \dot{s}=U(t)+f(t)+H(t)-\ddot{\eta}_{r}\end{array}\right.$

If all parameters of the robot system given in (19) are completely known, we can propose the following controller:

$U(t)=-\tilde{J}^{T}\left(\eta_{1}, \hat{\gamma}_{k}\right)\left(k_{1} e_{x}+k_{2} \dot{\hat{e}}_{x}+k_{3} \hat{s}_{x}\right)+\ddot{\eta}_{r}-f(t)-H(t)-\varepsilon(t)$

with:

$\hat{s}_{x}=Q\left(\eta_{1}, \eta_{2}\right) \hat{\gamma}_{k}-\dot{x}_{r}$

and $k_{1}, k_{2}$ and $k_{3} \in R^{6 \times 6}$ being diagonal positive matrices. $\hat{J}^{T}\left(\eta_{1}, \hat{\gamma}_{k}\right)$ is the estimated Jacobian transpose based on feedback of Cartesian tracking. The adaptation laws are updated by

$\dot{\hat{\gamma}}_{k}=k_{f}^{-1} \phi^{T}(t) k_{2}\left(\phi(t) \hat{\gamma}_{k}-z\right)+k_{f}^{-1} Q^{T}\left(\eta_{1}, \eta_{2}\right)\left(k_{1}+\beta k_{2}\right) e_{x}$

where $k_{f} \in R^{6 \times 6}$ is a diagonal positive matrix. Since $H(t)$ is uncertain, that may influence the performance of robot tracking. So, if Assumption 3 is verified, it is possible to use Time Delay Estimation [42]. In such case, the designed controller is given such that:

$U(t)=-\hat{J}^{T}\left(\eta_{1}, \hat{\gamma}_{k}\right)\left(k_{1} e_{x}+k_{2} \dot{\hat{e}}_{x}+k_{3} \hat{s}_{x}\right)+\ddot{\eta}_{r}-f(t)-\hat{H}(t)-\hat{\varepsilon}(t)$

where $\hat{H}(t)$ is obtained using TDE [42], using (3) to obtain:

$\hat{H}(t) \cong H\left(t-t_{d}\right)=\dot{\eta}_{2}\left(t-t_{d}\right)-f\left(t-t_{d}\right)-U\left(t-t_{d}\right)$

where $t_{d}$ is a positive constant assumed to be very small. Practically, the smallest constant can be reached is the sampling time. However, due to noisy measurements and nonlinearity of signals along the sampling time, a time delay error (TDR) $\varepsilon(t)$ exists, which would deteriorate the robustness and the accuracy of the robot. Unfortunately, the TDR is not available. In this case, let us apply an iterative estimator to estimate the TDR and to reduce its effect, and give to the control system more flexibility to deal with parameters' variation and unexpected disturbances. The iterative estimator is given as:

$\hat{\varepsilon}(t)=\varepsilon\left(t-t_{d}\right)-k_{4} s$

$\hat{\varepsilon}(t)=0, k_{4}=k I_{7 \times 7} \forall t \in\left[-t_{d}, 0\right]$

where, $k$ is a positive constant. The proof of the above Eq. (25) is given in Appendix A. The closed loop of the global system can be written using (19) and control input (23) as follows:

$\left\{\begin{array}{l}s=\dot{\eta}_{1}-\dot{\eta}_{r} \\ \dot{s}=-\hat{J}^{T}\left(\eta_{1}, \hat{\gamma}_{k}\right)\left(k_{1} e_{x}+k_{2} \dot{\hat{e}}_{x}+k_{3} \hat{s}_{x}\right)-\tilde{\varepsilon}(t)\end{array}\right.$

with: $\tilde{\varepsilon}=\hat{\varepsilon}(t)-\varepsilon(t)$ and $\varepsilon(t)=H(t)-\hat{H}(t)$ are respectively Time Delay Error, and dynamic uncertainties. Let us know state the maine result of the paper.

Theorem 1. The control law for sliding mode with time delay estimation (TDE) of uncertain robot dynamics determined in Eq. (23) ensures 
the asymptotic stability of the robot system. The desired torque input is given as:

$\tau=M_{0}\left(\eta_{1}\right) U(t)$

where $U(t)$ is given in (23).

Proof. To facilitate the proof of stability, let us define the term of the iterative estimator. First, we can write $\frac{d}{d t} \int_{t-t_{d}}^{t} \tilde{\varepsilon}^{T}(w) \tilde{\varepsilon}(w) d w$ as follows:

$\frac{d}{d t} \int_{t-t_{d}}^{t} \tilde{\varepsilon}^{T}(w) \tilde{\varepsilon}(w) d w=\tilde{\varepsilon}^{T}(t) \tilde{\varepsilon}(t)-\tilde{\varepsilon}^{T}\left(t-t_{d}\right) \tilde{\varepsilon}\left(t-t_{d}\right)$

Additionally,

$\frac{1}{2 k} \tilde{\varepsilon}^{T}(t) \tilde{\varepsilon}(t)-\frac{1}{2 k} \tilde{\varepsilon}^{T}\left(t-t_{d}\right) \tilde{\varepsilon}\left(t-t_{d}\right)=\tilde{\varepsilon}^{T}(t) s-s^{T} \frac{k_{4}^{T}}{2} s$

The details of Eqs. (28) and (29) are given in Appendix A. Consider the following Lyapunov function candidate:

$$
\begin{aligned}
V= & \frac{1}{2} s^{T} s+\frac{1}{2} e_{x}^{T}\left(k_{1}+\beta k_{2}\right) e_{x}+\frac{1}{2} \Delta \gamma_{k}^{T} k_{f} \Delta \gamma_{k} \\
& +\frac{1}{2 k} \int_{t-t_{d}}^{t} \tilde{\varepsilon}^{T}(w) \tilde{\varepsilon}(w) d w
\end{aligned}
$$

with: $\Delta \gamma_{k}=\gamma_{k}-\hat{\gamma}_{k}, \tilde{\varepsilon}=\hat{\varepsilon}(t)-\varepsilon(t)$ and $\varepsilon(t)=H(t)-\hat{H}(t)$ are respectively the estimation errors of kinematic uncertainties, Time Delay Error, and dynamic uncertainties. The derivative of the proposed Lyapunov function with respect to time is obtained as:

$$
\begin{aligned}
\dot{V}= & s^{T} \dot{s}+e_{x}^{T}\left(k_{1}+\beta k_{2}\right) \dot{e}_{x}-\Delta \gamma_{k}^{T} k_{f} \dot{\hat{\gamma}}_{k}+\frac{1}{2 k} \tilde{\varepsilon}^{T}(t) \tilde{\varepsilon}(t) \\
& -\frac{1}{2 k} \tilde{\varepsilon}^{T}\left(t-t_{d}\right) \tilde{\varepsilon}\left(t-t_{d}\right)
\end{aligned}
$$

Substituting $\dot{s}$ from (19) and using (29) into (31), we find:

$$
\begin{aligned}
\dot{V}= & -s^{T} \hat{J}^{T}\left(\eta_{1}, \hat{\gamma}_{k}\right)\left(k_{1} e_{x}+k_{2} \dot{\hat{e}}_{x}+k_{3} \hat{s}_{x}\right)+e_{x}^{T}\left(k_{1}+\beta k_{2}\right) \dot{e}_{x} \\
& -\Delta \gamma_{k}^{T} k_{f} \dot{\hat{\gamma}}_{k}+s^{T} \varepsilon(t)-s^{T} \hat{\varepsilon}(t)+\tilde{\varepsilon}^{T}(x, t) s-s^{T} \frac{k_{4}^{T}}{2} s \\
= & -s^{T} \hat{J}^{T}\left(\eta_{1}, \hat{\gamma}_{k}\right)\left(k_{1} e_{x}+k_{2} \dot{\hat{e}}_{x}+k_{3} \hat{s}_{x}\right)+e_{x}^{T}\left(k_{1}+\beta k_{2}\right) \dot{e}_{x} \\
& -\Delta \gamma_{k}^{T} k_{f} \dot{\hat{\gamma}}_{k}-s^{T} \frac{k_{4}^{T}}{2} s
\end{aligned}
$$

Substituting (17) and (22) into (32), we find:

$$
\begin{aligned}
\dot{V}= & -\hat{s}_{x}^{T} k_{1} e_{x}-\hat{s}_{x}^{T} k_{2} \dot{\hat{e}}_{x}-\hat{s}_{x}^{T} k_{3} \hat{s}_{x}-s^{T} \frac{k_{4}^{T}}{2} s+e_{x}^{T}\left(k_{1}+\beta k_{2}\right) \dot{e}_{x} \\
& -\Delta \gamma_{k}^{T} \phi^{T}(t) k_{2} \phi(t) \Delta \gamma_{k}-\Delta \gamma_{k}^{T} Q^{T}\left(\eta_{1}, \eta_{2}\right)\left(k_{1}+\beta k_{2}\right) e_{x}
\end{aligned}
$$

We have from (5), (10) and (13):

$\hat{s}_{x}=\dot{e}_{x}+\beta e_{x}-Q\left(\eta_{1}, \eta_{2}\right) \Delta \gamma_{k}=\dot{\hat{e}}_{x}+\beta e_{x}$

with:

$Q\left(\eta_{1}, \eta_{2}\right) \Delta \gamma_{k}=J\left(\eta_{1}\right) \eta_{2}-\hat{J}\left(\eta_{1}, \hat{\gamma}_{k}\right) \eta_{2}=\dot{x}-\dot{\hat{x}}$

From (34) we have:

$\dot{\hat{e}}_{x}=\dot{e}_{x}-Q\left(\eta_{1}, \eta_{2}\right) \Delta \gamma_{k}$

Substituting (34) and (36) into (33), we find:

$$
\begin{aligned}
\dot{V}= & -\dot{e}_{x}^{T} k_{1} \dot{e}_{x}+2 \dot{e}_{x}^{T} k_{2} Q\left(\eta_{1}, \eta_{2}\right) \Delta \gamma_{k}-\beta e_{x}^{T} k_{1} e_{x}-\hat{s}_{x}^{T} k_{3} \hat{s}_{x}-s^{T} \frac{k_{4}^{T}}{2} s \\
& -\Delta \gamma_{k}^{T} \phi^{T}(t) k_{2} \phi(t) \Delta \gamma_{k}-\Delta \gamma_{k}^{T} Q^{T}(\theta, \dot{\theta}) k_{2} Q\left(\eta_{1}, \eta_{2}\right) \Delta \gamma_{k}
\end{aligned}
$$

While $\dot{\hat{e}}_{x}=\dot{e}_{x}-Q\left(\eta_{1}, \eta_{2}\right) \Delta \gamma_{k}$, (37) can be reduced to

$$
\dot{V}=-\dot{\hat{e}}_{x}^{T} k_{1} \dot{\hat{e}}_{x}-\beta e_{x}^{T} k_{1} e_{x}-\hat{s}_{x}^{T} k_{3} \hat{s}_{x}-s^{T} \frac{k_{4}^{T}}{2} s-\Delta \gamma_{k}^{T} \phi^{T}(t) k_{2} \phi(t) \Delta \gamma_{k}
$$

From (30), we can easily see that $V$ is positive definite in $s, \dot{\hat{e}}_{x}, \Delta \gamma_{k}$ and $\tilde{\varepsilon}(t)$. Since $\dot{V}$ is negative definite from (38), and $V$ is bounded, this implies that $s, e_{x}, \hat{\gamma}_{k}$ and $\hat{\varepsilon}(t)$ are bounded. From (17) $\hat{s}_{x}$ is bounded because $s$ is bounded. $x$ is bounded because $x_{d}$ is assumed bounded (Assumption 4). While $e_{x}$ is bounded and $\dot{x}_{d}$ is bounded (Assumption 4), this implies $\dot{x}_{r}$ in (10) is bounded. The pseudo-inverse of the Jacobian matrix in (15) is non singular and bounded (all joints of manipulator are revolute), this means that $\dot{\theta}_{r}$ is bounded. We observe from (17) that $\dot{\theta}$ is bounded, this implies $\dot{x}$ is bounded while the Jacobian matrix is bounded. We remark from (11) that $\ddot{x}_{r}$ is bounded because $\dot{e}_{x}$ and $\ddot{x}_{d}$ are bounded (Assumption 4). It is clear from (16) that $\ddot{\theta}_{r}$ is bounded. We see from (18) that $\dot{s}$ is bounded, that implies $\ddot{\theta}$ is bounded. We can conclude from (14) that $\dot{\hat{S}}_{x}$ is bounded. The derivative of (34) is expressed as follows:

$\dot{\hat{s}}_{x}=\ddot{\hat{e}}_{x}+\beta \dot{e}_{x}$

where $\ddot{\hat{e}}_{x}=\ddot{\hat{x}}-\ddot{x}_{d}$ is bounded. Since $V$ is bounded and $\dot{V}$ is continuous and negative semidefinite, we can utilize Barbalat's lemma by differentiating Eq. (38) such that

$$
\begin{aligned}
\ddot{V}= & -2 \dot{\hat{e}}_{x}^{T} k_{1} \ddot{\hat{e}}_{x}-2 \beta e_{x}^{T} k_{1} \dot{e}_{x}-2 \hat{s}_{x}^{T} k_{3} \dot{\hat{S}}_{x}-2 s^{T} \frac{k_{4}^{T}}{2} \dot{s} \\
& -\Delta \gamma_{k}^{T} \phi^{T}(t) k_{2}\left(\dot{\phi}(t) \Delta \gamma_{k}-\phi(t) \dot{\hat{\gamma}}_{k}\right)
\end{aligned}
$$

Since $\dot{\theta}$ and $\ddot{\theta}$ are bounded, this means $\phi(t), \dot{\phi}(t)$ are bounded. This proves that $\ddot{V}$ is bounded since $e_{x}, \dot{e}_{x}, \dot{\hat{e}}_{x}, \ddot{\hat{e}}_{x}, \dot{\hat{S}}_{x}, \hat{\gamma}_{k}$ are all bounded. So, $\dot{V}$ is continuous and negative semidefinite; according to Barbalat's lemma. we have $e_{x} \rightarrow 0, \dot{\hat{e}}_{x} \rightarrow 0, s \rightarrow 0$ and $\phi(t) \Delta \gamma_{k} \rightarrow 0$ as $t \rightarrow \infty$. Now, the derivative of Eq. (34) with repect to time yields:

$\ddot{e}_{x}+\beta \dot{e}_{x}=\dot{\hat{S}}_{x}+\dot{Q}(\theta, \dot{\theta}) \Delta \gamma_{k}-Q(\theta, \dot{\theta}) \dot{\hat{\gamma}}_{k}$

That means $\ddot{e}_{x}$ is also bounded. In this case, we have $\dot{e}_{x} \rightarrow 0$ as $t \rightarrow \infty$ since $\ddot{e}_{x}$ and $e_{x}$ are bounded. Hence, the proof is complete. The lock diagram of the proposed controller is given in Fig. 2 .

\section{Experimental and comparative Study}

\subsection{Experiment set-up}

The experimental setup of the proposed system is shown in Fig. 3. The system consists of three processing units. The first is a PC from where the top-level commands are sent to the robot using LabVIEW interface, i.e. the control scheme selection, joint or Cartesian space trajectory, gain adjustments, etc. This PC also receives the data after the robot task is executed to analyze its performance. The other two processing units are part of a National Instruments PXI platform. Firstly, a NI-PXI 8081 controller card with an Intel Core Duo processor; in this card, the main operating system of the robot and the top-level control scheme are executed. In our case, the sliding mode based controller as well as the esti-

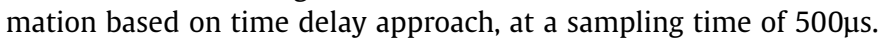
The inverse kinematics algorithm also runs inside this control loop. Finally, at input-output level, a NI PXI-7813R remote input-output card with an FPGA (field programmable gate array) executes the low-level control; i.e. a PI current control loop (sampling time of $50 \mu \mathrm{s})$ to maintain the current of the motors required by the main controller. Also, in this FPGA, the position feedback (Cartesian and joint) via Hall-sensors (joint position) and Kinect (Cartesian Endeffector position), basic input-output tasks are executed.

The modified Denavit-Hartenberg ( $\mathrm{DH}$ ) parameters are given in Table 1 . These parameters are obtained from reference frames as shown in Fig. 1, and are used to obtain the homogeneous transformation matrices. 


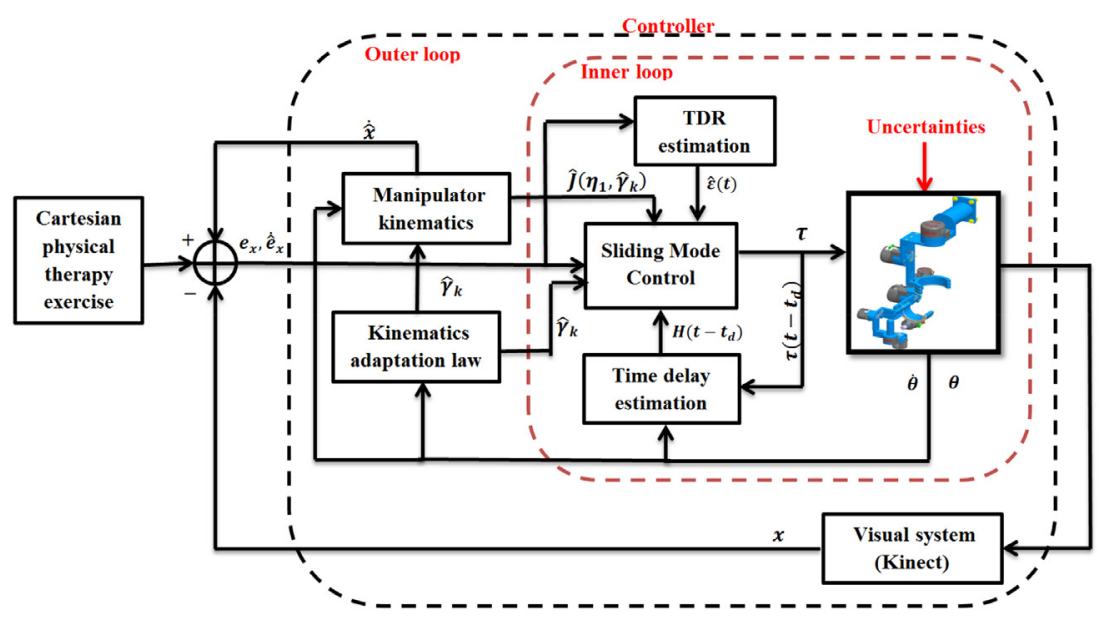

Fig. 2. Block diagram of the proposed controller for exoskeleton robot with unknown kinematics/dynamics.

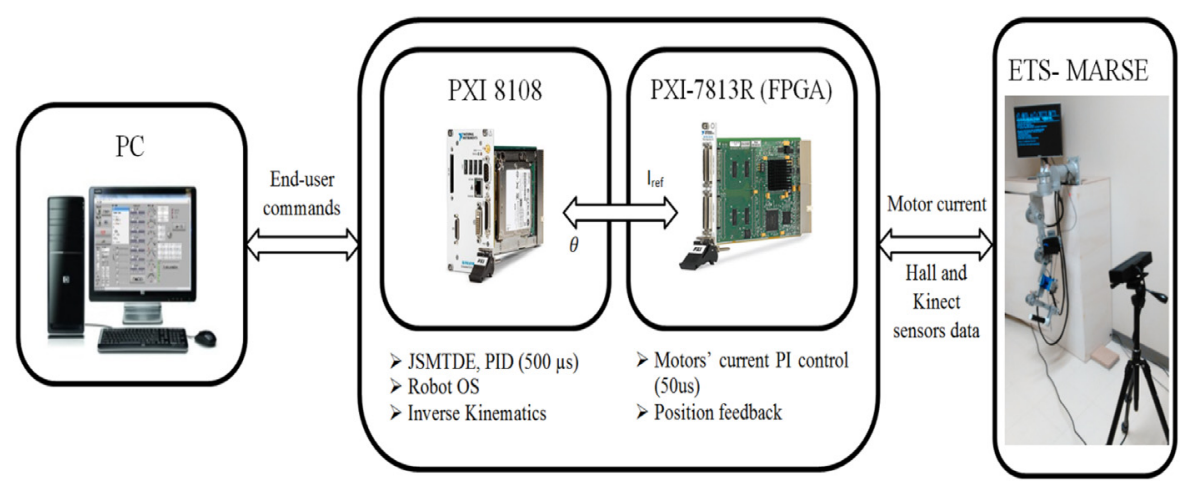

Fig. 3. Experiments architecture.

Table 1

Modified Denavit-Hartenberg parameters.

\begin{tabular}{lllll}
\hline Joint $(i)$ & $\alpha_{i-1}$ & $a_{i-1}$ & $d_{i}$ & $\theta_{i}$ \\
\hline 1 & 0 & 0 & $d_{s}$ & $\theta_{1}$ \\
2 & $-\pi / 2$ & 0 & 0 & $\theta_{2}$ \\
3 & $\pi / 2$ & 0 & $d_{e}$ & $\theta_{3}$ \\
4 & $-\pi / 2$ & 0 & 0 & $\theta_{4}$ \\
5 & $\pi / 2$ & 0 & $d_{w}$ & $\theta_{5}$ \\
6 & $-\pi / 2$ & 0 & 0 & $\theta_{6}-\pi / 2$ \\
7 & $-\pi / 2$ & 0 & 0 & $\theta_{7}$ \\
\hline
\end{tabular}

Table 3

Physical parameters of ETS-MARSE.

\begin{tabular}{llll}
\hline Joint $(\mathrm{i})$ & Mass $(\mathrm{Kg})$ & Centre of mass $(\mathrm{m})$ & Link length $(\mathrm{m})$ \\
\hline 1 & 3.475 & 0.0984 & 0.145 \\
2 & 3.737 & 0.1959 & 0 \\
3 & 0 & 0 & 0.25 \\
4 & 2.066 & 0.163 & 0 \\
5 & 0 & 0 & 0.267 \\
6 & 0.779 & 0.121 & 0 \\
7 & 0.496 & 0.0622 & 0
\end{tabular}

Table 2

Workspace ETS-MARSE.

\begin{tabular}{lll}
\hline Joints & Motion & Workspace \\
\hline 1 & Shoulder joint horizontal flexion/extension & $0^{\circ} / 140^{\circ}$ \\
2 & Shoulder joint vertical flexion/extension & $140^{\circ} / 0^{\circ}$ \\
3 & Shoulder joint internal/external rotation & $-85^{\circ} / 75^{\circ}$ \\
4 & Elbow joint flexion/extension & $120^{\circ} / 0^{\circ}$ \\
5 & Forearm joint pronation/supination & $-85^{\circ} / 85^{\circ}$ \\
6 & Wrist joint ulnar/radial deviation & $-30^{\circ} / 20^{\circ}$ \\
7 & Wrist joint flexion/extension & $-50^{\circ} / 60^{\circ}$ \\
\hline
\end{tabular}

The workspace of the designed robot is given in Table 2 and the physical parameters of ETS-MARSE relative to the base reference frame are given in Table 3. The details of the parameters and design of ETS- MARSE are given in [35].

In the experiments, the desired Cartesian trajectory corresponds to a prescribed passive physical therapy task performed by three healthy subjects (age: $27 \pm 4.6$ years; height: $170 \pm 8.75 \mathrm{~cm}$;

weight: $75 \pm 18 \mathrm{Kg}$ ). This trajectory (Initial position $\rightarrow$ Target$\mathrm{A} \rightarrow$ Target-B $\rightarrow$ Target- $\mathrm{C} \rightarrow$ Initial position) is expressed in Cartesian space to evaluate the proposed control. In this case, the position of the Cartesian End-Effector of the robot is provided by visual system (Kinect). For the carried object, the subject-robot system carried an object with unknown weight and dimensions during the desired trajectory. It is important to notice that the external disturbances here are represented by different physiological conditions of the subjects, such as non-linear biomechanical characteristics of the musculoskeletal system, the different weight of the upper-limb for each subject, the presence of spasticity in neurological patients, etc.

The experimental control gains are chosen by trial and error as follows: $k_{1}=20 I_{6 \times 6}, \quad k_{2}=70 I_{6 \times 6}, k_{3}=18 I_{7 \times 7} \quad k_{f}=$ $0.01 I_{6 \times 6}, k_{4}=0.5 I_{7 \times 7} \quad \beta=10$. The experimental results are given in Figs 4-6. 


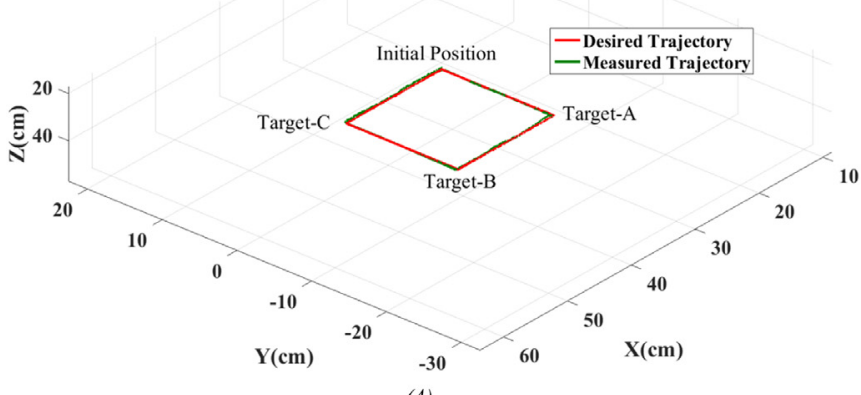

(A)
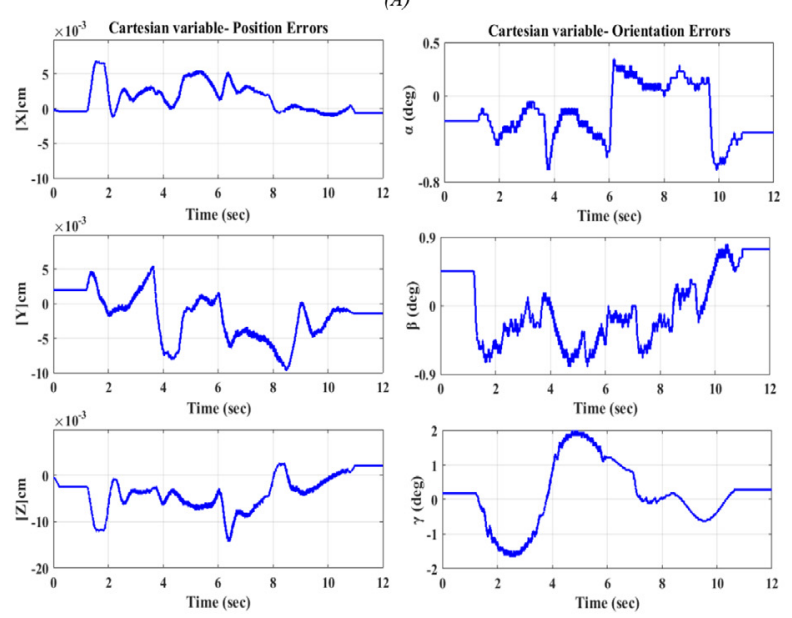

(B)

Fig. 4. (A) Workspace performance of the robot in Cartesian space performed by Subject-1: (age: 27 years; height: $177 \mathrm{~cm}$; weight: $83 \mathrm{Kg}$ ). (B) Cartesian errors.

\subsection{Experimental results}

\subsubsection{The main results of the proposed controller with recursive} control

The experimental results with ETS-MARSE robot in Cartesian space performed by Subject-1: (age: 27 years; height: $177 \mathrm{~cm}$; weight: $83 \mathrm{Kg}$ ) using the designed strategy are shown in Fig. 4. As we see in this figure (Fig. 4(A)), the desired trajectory (red line)

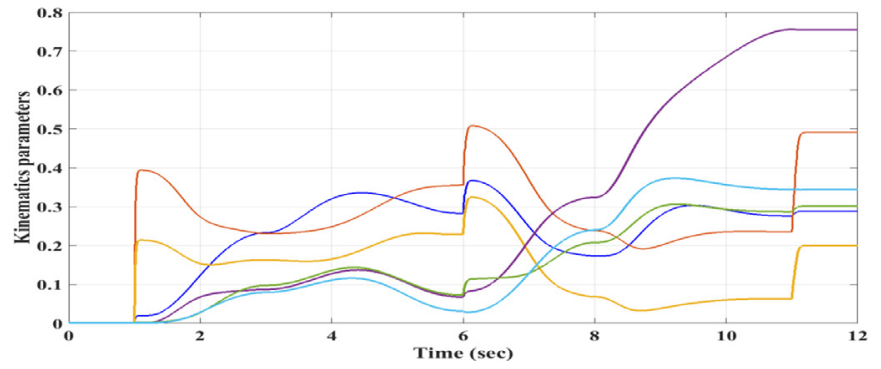

Fig. 6. Kinematic $\left(\hat{\gamma}_{k}\right)$ parameters convergence of the exoskeleton robot.

nearly overlapped with the measured trajectory (green line). It can be noticed that these results are fairly good. Fig. 4(B) presents the Cartesian errors as functions of time. From this figure, it is obvious that the Cartesian errors are getting smaller along the desired trajectory. Fig. 5 shows that the control input is bounded without any noticeable control chattering. Finally, the convergence of the kinematic $\left(\hat{\gamma}_{k}\right)$ and dynamic $(\hat{H}(t))$ parameters of the exoskeleton robot during the proposed control is shown in Fig. 6 and 7 respectively. These results confirm that the control strategy is able to achieve the desired robot's performance even if the nonlinear kinematics and dynamics of the exoskeleton robot are uncertain and the parameters of Kinect (camera) device are not completely known.

4.2.2. The results of the proposed controller without recursive control

Fig. 8(A) presents the workspace performance of the robot in Cartesian space (red is the desired trajectory, green is real trajectory) performed with performed by Subject-1: (age: 28 years; height: $177 \mathrm{~cm}$; weight: $83 \mathrm{Kg}$ ) using the proposed controller without a recursive controller. In fact, we remark from figures (Fig. 8 (A-B)) that the proposed controller without recursive controller shows a good performance. Where the Cartesian error (Fig. 8(B)) is getting smaller with time. However, the control inputs of the conventional approach, presented in Fig. 9, illustrate a noisy control input with a small chattering phenomenon, meanwhile, noise and peaks appear also in the estimation parameters of the unknown dynamics parameters as we see in Fig. 10. On the other hand, the proposed controller with recursive control provides a smooth control input (Fig. 5) and smooth estimation of unknown dynamics parameters (Fig. 7). Therefore, we can say that the TDR is the main cause of the noise in the control input, which may damage the motors. From the comparison of the two experimental results, we can
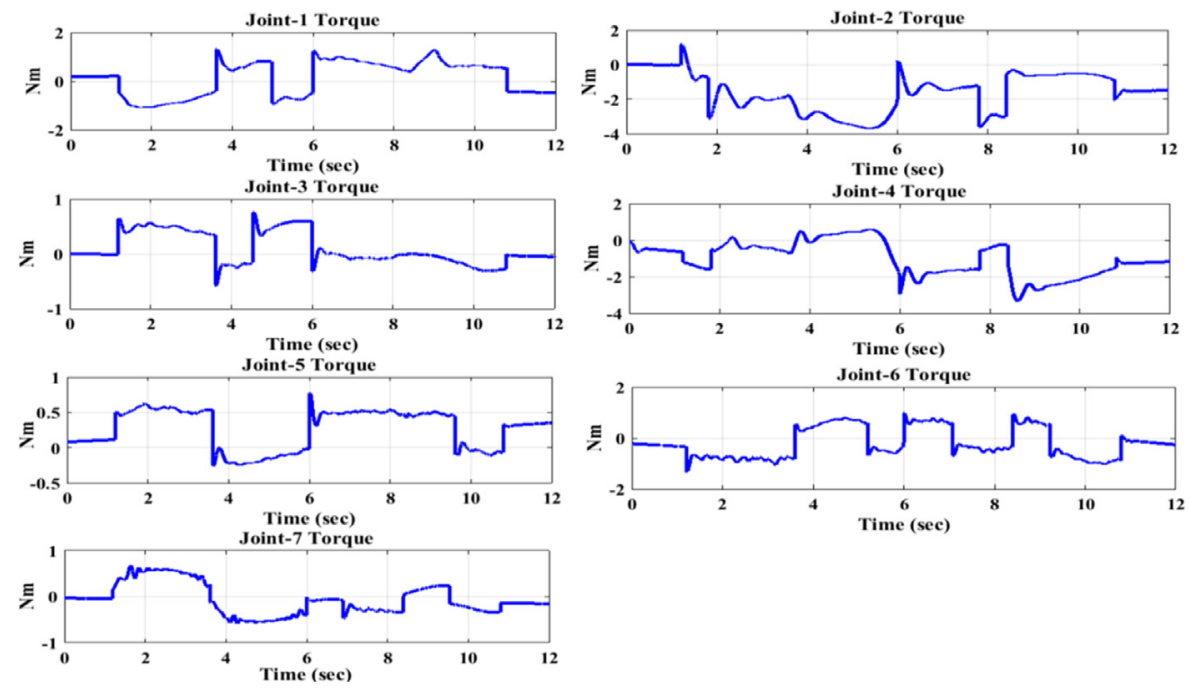

Fig. 5. Control input. 


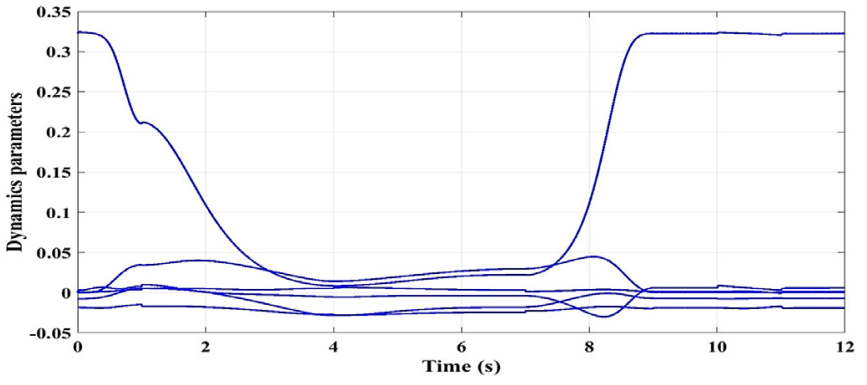

Fig. 7. Unknown Dynamics $(\hat{H}(t)$ parameters convergence of the exoskeleton robot.

conclude that the proposed strategy with a recursive action provides a high level of precision and robustness against the nonlinear dynamic uncertainties and unknown disturbances.

\subsection{Comparative study}

In order to evaluate the efficiency and feasibility of the proposed control scheme, we compared it experimentally with the conventional adaptive visual tracking control presented in [10]. This latter is characterized by more complex implementation due to the complex regressor dynamic matrix, while the robot had a high degree of freedom (7-DoFs). To compute the regressor dynamic matrix of the robot, we use the virtual decomposition control (VDC) presented in [31].

Fig. 11(A) presents the Cartesian trajectory tracking in the 3D workspace (red is the desired trajectory, green is real trajectory) performed by the same subject (Subject-1: age: 27 years; height: $177 \mathrm{~cm}$; weight: $83 \mathrm{Kg}$ ) using the conventional controller. It is clear from (Fig. 11 (A and B)) that the conventional controller provides a good tracking performance. Where, the error is converging along the desired trajectory as we show in Fig 11(B). Nevertheless, there is a presence of chattering phenomenon in the control inputs as shown in Fig 12, which may damage the motors of the robot. Compared with the smooth control input that is provided by the proposed strategy (Fig. 5), we can conclude that the proposed strategy is easily implementable and provides a high precision and robustness to the kinematic/dynamic uncertainties, with unknown disturbances, and uncertainties of the camera parameters.
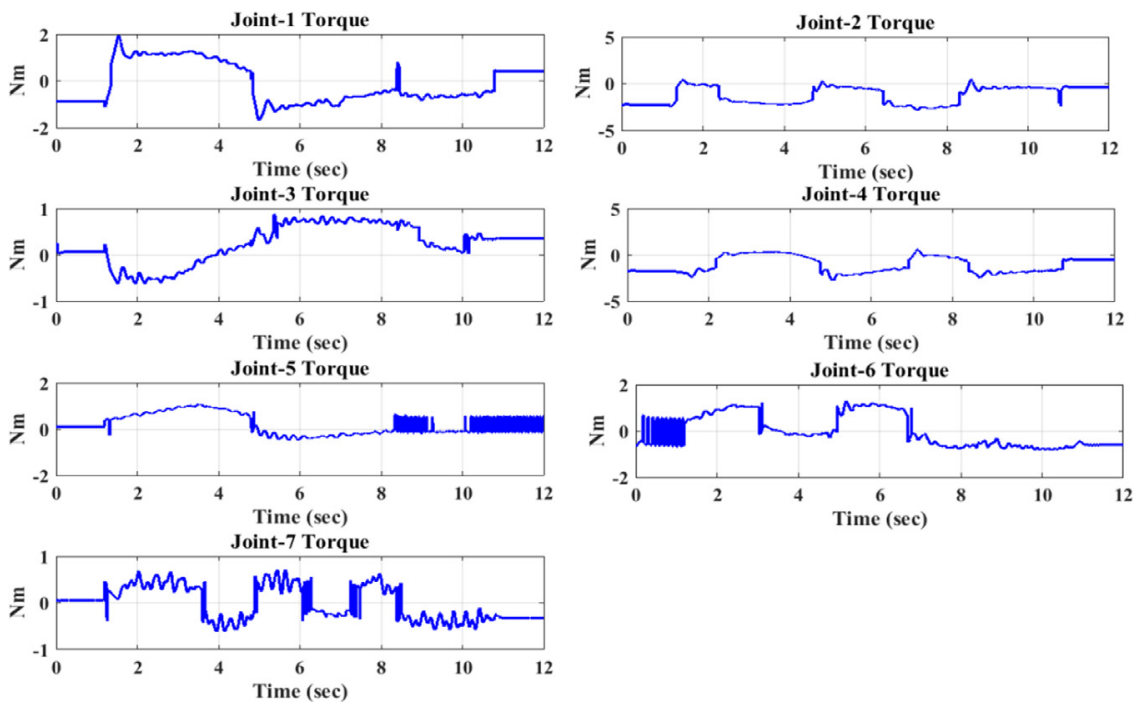

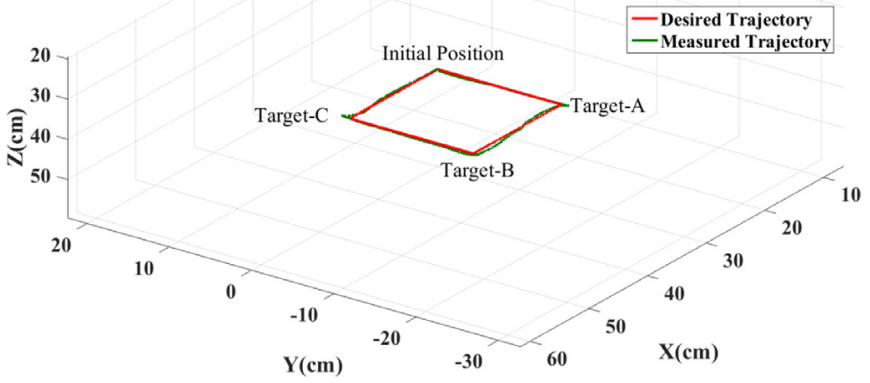

(A)
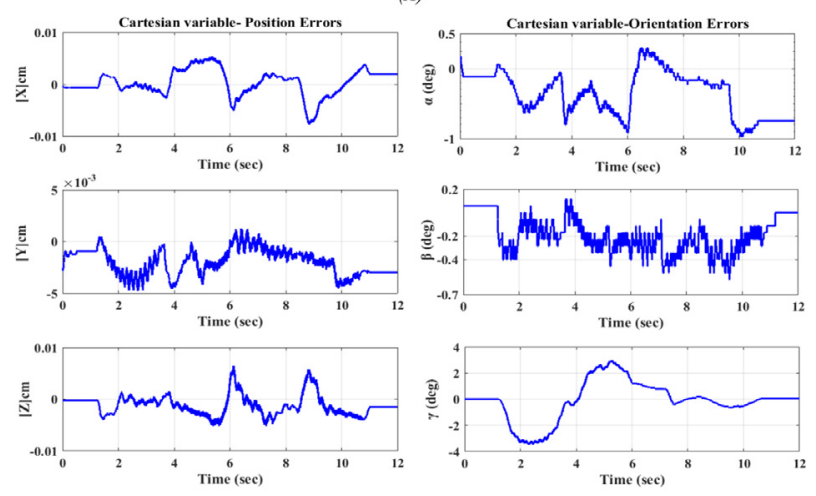

(B)

Fig. 8. (A) Workspace performance of the robot in Cartesian space performed by Subject-1: (age: 27 years; height: $177 \mathrm{~cm}$; weight: $83 \mathrm{Kg}$ ). (B) Cartesian errors.

To show more the feasibility of the designed strategy, we propose a numerical comparison between the above controller (conventional controller and proposed controller) by calculating the root mean square (RMS) of the error and the control input of each controller as follows $\|e\|_{\text {error }}=\sqrt{\frac{1}{N} \sum_{i=1}^{N}\|e\|^{2}}$ and $\|\tau\|_{\text {error }}=$ $\sqrt{\frac{1}{N} \sum_{i=1}^{N}\|\tau\|^{2}}$, where $\mathrm{N}$ is the number of the sampling time steps of the simulation. The evaluation of the controller is given Table 4 .

It confirms that the proposed approach gives the robot a high degree of efficiency for dealing well with parameter variations and

Fig. 9. Control input. 


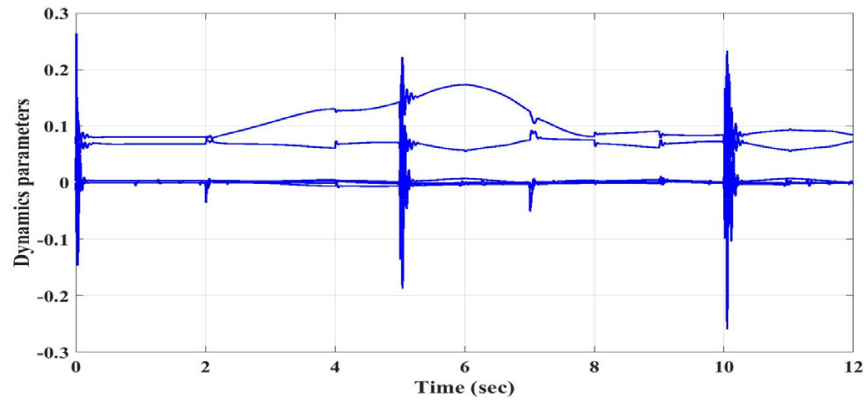

Fig. 10. Unknown Dynamic parameters convergence of the exoskeleton robot.

the nonlinear kinematic/dynamic uncertainties in presence of unknown disturbances (different subjects with different physiological conditions. These conditions include non-linear biomechanical characteristics of the musculoskeletal system, the different weight of the upper limb for each patient, the presence of spasticity/dystonia, muscle weakness in neurological patients,... etc) and parameters' uncertainties of the Kinect compared with the conventional adaptive controller. The proposed controller provides consistent performance with different subjects, keeping the RMS error and general torque input at a small value compared with the conventional controller. Compared with similar tests performed in a previous study with ETS-MARSE robot, the proposed control based on TDE presents an excellent performance as the Virtual Decomposition Control [31], and better than PID and Computed Torque Control (CTC)[31].

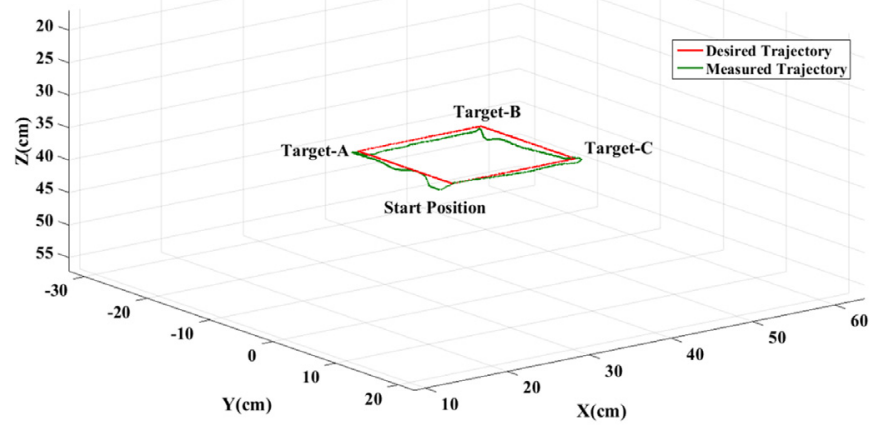

(A)
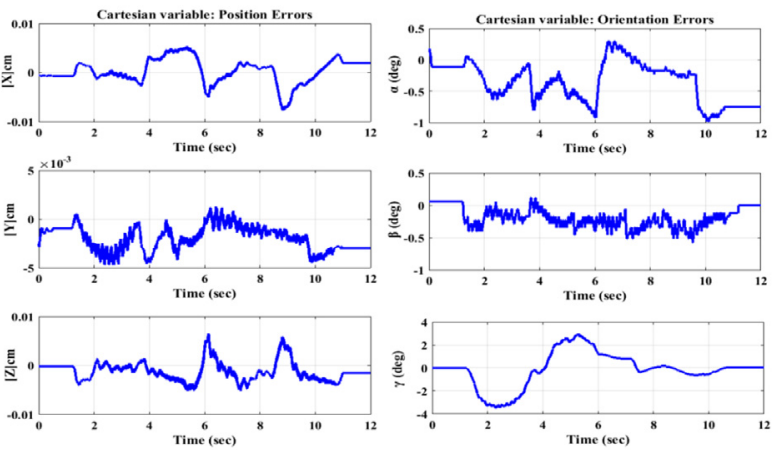

(B)

Fig. 11. (A) Workspace performance of the robot in Cartesian space performed by Subject-1: (age: 27 years; height: $177 \mathrm{~cm}$; weight: $83 \mathrm{Kg}$ ). (B) Cartesian errors.
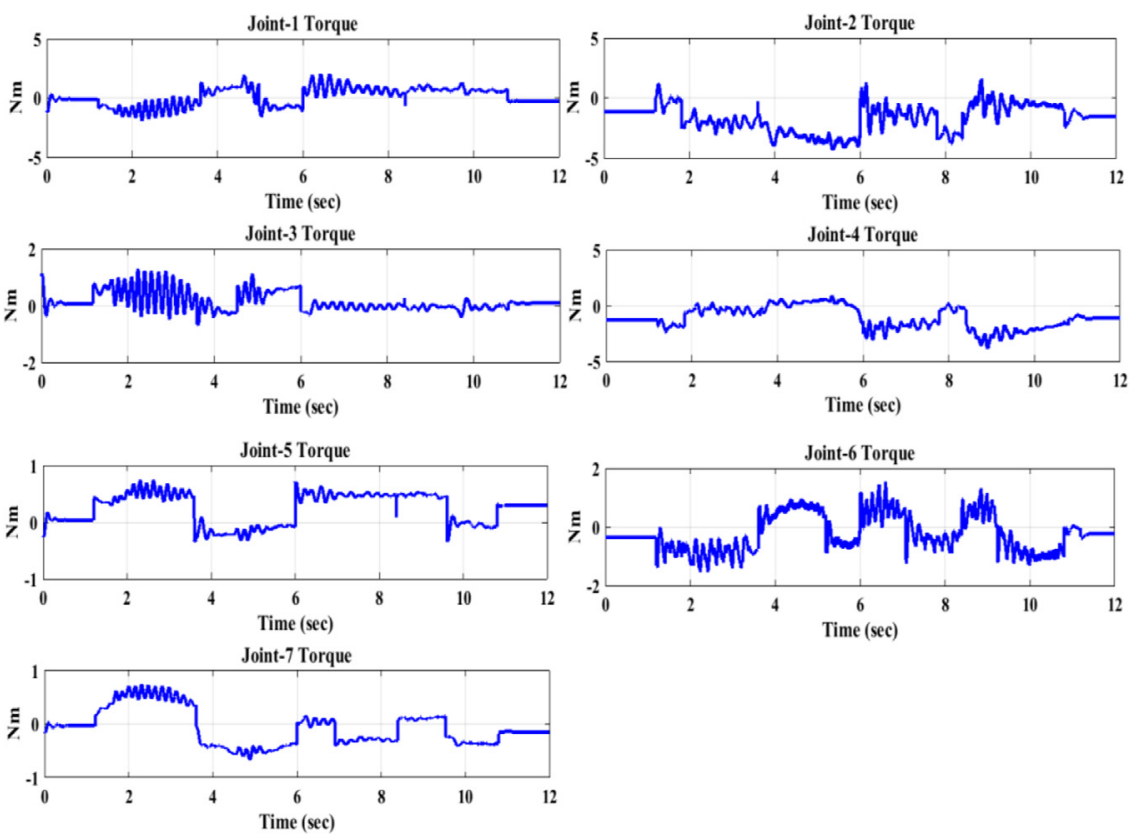

Fig. 12. Control input.

Table 4

Comapative study.

\begin{tabular}{|c|c|c|c|c|c|c|}
\hline \multirow[t]{3}{*}{ Subjects } & \multicolumn{6}{|c|}{ Root mean square (RMS) } \\
\hline & \multicolumn{2}{|c|}{ The proposed controller with recursive action } & \multicolumn{2}{|c|}{ The proposed controller without recursive action } & \multicolumn{2}{|c|}{ Conventional controller } \\
\hline & 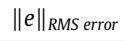 & $\|\tau\|_{\text {RMS Torque }}$ & 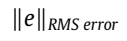 & $\|\tau\|_{\text {RMS Torque }}$ & 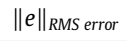 & $\|\tau\|_{\text {RMS Torque }}$ \\
\hline Subject-1 & 0.0317 & 2.0015 & 0.0487 & 2.6887 & 0.1987 & 4.8897 \\
\hline Subject-2 & 0.0299 & 1.8708 & 0.0417 & 2.1748 & 0.1478 & 4.1766 \\
\hline Subject-3 & 0.0281 & 1.9501 & 0.0335 & 2.8874 & 0.1797 & 4.7468 \\
\hline
\end{tabular}




\section{Conclusion}

In this paper, we proposed an adaptive control of a 7DOFs exoskeleton robot with uncertain kinematics and dynamics based on sliding mode controller. Estimated Jacobian matrix is taken into consideration. The control strategy is achieved by inner/outer loops, where the outer loop is designed to estimate the nonlinear kinematic parameters and the Inner loop is designed to estimate the unknown dynamics of the robot using TDE approach and recursive control to limit the effect of its time delay error. The main benefit of the proposed adaptive control approach is that precise knowledge of the kinematic/dynamic parameters of the robot is not mandatory. Where, the proposed adaptive strategy is characterized by the ease of real-time implementation and provides a high precision and robustness to the kinematic/dynamic uncertainties, unknown perturbation, and uncertainties of the camera parameters. Additionally, the time delay error is taken into account to improve the accuracy of the robot performance. The stability analysis of inner/outer visual tracking control with kinematics/dynamics uncertainties taken into consideration the uncertainties in the camera device was proved by the Lyapunov function theorem. The robustness of the proposed control was proved with a Cartesian functional therapy task performed by the ETS-MARSE robot. The experimental results show the effectiveness, facility of implementation and accuracy of the proposed approach.

\section{Appendix A}

1. Proof of Eqs. (18) and (19) [6, 4]: for very small sampling time period, it is acceptable to assume that the previous time delay error equals the current time delay error. In this case, considering:

$\left\{\begin{array}{l}\varepsilon(t)=\varepsilon\left(t-t_{d}\right) \\ \tilde{\varepsilon}(t)=\varepsilon(t)-\hat{\varepsilon}(t) \\ \hat{\varepsilon}(t)=\hat{\varepsilon}\left(t-t_{d}\right)+g(t)\end{array}\right.$

where $g(t) \in R$ is a vector to be defined later. Then

$\frac{d}{d t} \int_{t-t_{d}}^{t} \tilde{\varepsilon}^{T}(w) \tilde{\varepsilon}(w) d w=-2 \tilde{\varepsilon}^{T}(t) g(t)-g^{T}(t) g(t)$

Proof. It is easy to conclude that:

$\frac{d}{d t} \int_{t-t_{d}}^{t} \tilde{\varepsilon}^{T}(w) \tilde{\varepsilon}(w) d w=\tilde{\varepsilon}^{T}(t) \tilde{\varepsilon}(t)-\tilde{\varepsilon}^{T}\left(t-t_{d}\right) \tilde{\varepsilon}\left(t-t_{d}\right)$

Considering the following equation $\hat{\varepsilon}(t)=\hat{\varepsilon}\left(t-t_{d}\right)+g(t)$ :

$\tilde{\varepsilon}^{T}\left(t-t_{d}\right) \tilde{\varepsilon}\left(t-t_{d}\right)$

$=\left[\varepsilon^{T}\left(t-t_{d}\right)-\hat{\varepsilon}^{T}\left(t-t_{d}\right)\right]\left[\varepsilon\left(t-t_{d}\right)-\hat{\varepsilon}\left(t-t_{d}\right)\right]$

$=\left[\varepsilon^{T}(t)-\hat{\varepsilon}^{T}(t)+g^{T}(t)\right][\varepsilon(t)-\hat{\varepsilon}(t)+g(t)]$

$=\tilde{\varepsilon}^{T}(t) \tilde{\varepsilon}(t)+2 \tilde{\varepsilon}^{T}(t) g(t)+g^{T}(t) g(t)$

Thus, we can obtain:

$\tilde{\varepsilon}^{T}(t) \tilde{\varepsilon}(t)-\tilde{\varepsilon}^{T}\left(t-t_{d}\right) \tilde{\varepsilon}\left(t-t_{d}\right)=-2 \tilde{\varepsilon}^{T}(t) g(t)-g^{T}(t) g(t)$

Let us choosing $g(t)$ such that:

$g(t)=-k_{4} S$

Substituting Eq. (47) in Eq. (46), we obtain:

$\tilde{\varepsilon}^{T}(t) \tilde{\varepsilon}(t)-\tilde{\varepsilon}^{T}\left(t-t_{d}\right) \tilde{\varepsilon}\left(t-t_{d}\right)=2 \tilde{\varepsilon}^{T}(t) k_{4} S-\left(k_{4} S\right)^{T}\left(k_{4} s\right)$

Hence, we have:

$\frac{1}{2 k} \tilde{\varepsilon}^{T}(t) \tilde{\varepsilon}(t)-\frac{1}{2 k} \tilde{\varepsilon}^{T}\left(t-t_{d}\right) \tilde{\varepsilon}\left(t-t_{d}\right)=\tilde{\varepsilon}^{T}(x, t) s-s^{T} \frac{k_{4}^{T}}{2} s$

\section{References}

[1] S. Arimoto, Robotics research toward explication of everyday physics, Int. J Robot. Res. 18 (1999) 1056-1063.

[2] Y. Bai, J.D. Biggs, F.B. Zazzera, N. Cui, Adaptive attitude tracking with active uncertainty rejection, J. Guid. Control Dyn. 41 (2017) 1-9.

[3] S. Balasubramanian, R. Wei, M. Perez, B. Shepard, E. Koeneman, J. Koeneman, et al., RUPERT: an exoskeleton robot for assisting rehabilitation of arm functions, in: Proceedings of 2008 Virtual Rehabilitation, 2008, pp. 163-167.

[4] B. Brahim, S. Maarouf, C.O. Luna, B. Abdelkrim, M.H. Rahman, Adaptive iterative observer based on integral backstepping control for upper extremity exoskelton robot, in: Proceedings of 8th International Conference on Modelling Identification and Control (ICMIC), 2016, pp. 886-891.

[5] A. Brahmi, M. Saad, G. Gauthier, B. Brahmi, W.-H. Zhu, and J. Ghommam, Adaptive backstepping control of mobile manipulator robot based on virtual decomposition approach, in: Proceedings of the 8th International Conference on Modelling, Identification and Control (ICMIC), pp. 707-712.

[6] B. Brahmi, M.H. Rahman, M. Saad, C.O. Luna, Iterative estimator-based nonlinear backstepping control of a robotic exoskeleton, Int. J. Mech. Aerosp. Ind., Mechatron. Manuf. Eng. 10 (2016) 1202-1208.

[7] B. Brahmi, M. Saad, C.O. Luna, P. Archambault, M. Rahman, Sliding mode control of an exoskeleton robot based on time delay estimation, in: Proceedings of International Conference on Virtual Rehabilitation ICVR, 2017, pp. 1-2.

[8] B. Brahmi, M. Saad, C. Ochoa-Luna, M.H. Rahman, Adaptive control of an exoskeleton robot with uncertainties on kinematics and dynamics, in: Proceedings of International Conference on Rehabilitation Robotics (ICORR), 2017, pp. 1369-1374.

[9] B. Brahmi, M. Saad, M.H. Rahman, C. Ochoa-Luna, Cartesian trajectory tracking of a 7-DOF exoskeleton robot based on human inverse kinematics, IEEE Trans. Syst., Man Cybern. Syst. PP (2017) 1-12.

[10] C.C. Cheah, Approximate Jacobian control for robot manipulators, Advances in Robot Control, Springer, 2006, pp. 35-53.

[11] C.C. Cheah, S. Kawamura, S. Arimoto, Feedback control for robotic manipulator with an uncertain Jacobian matrix, J. Robot. Syst. 16 (1999) 119-134.

[12] C.-C. Cheah, C. Liu, J.-J.E. Slotine, Adaptive Jacobian tracking control of robots based on visual task-space information, in: Proceedings of the 2005 IEEE International Conference on Robotics and Automation, 2005, pp. 3498-3503.

[13] C.-C. Cheah, C. Liu, J.-J.E. Slotine, Adaptive tracking control for robots with unknown kinematic and dynamic properties, Int. J. Robot. Res. 25 (2006) 283-296.

[14] A. De Morand, Pratique de la rééducation neurologique, Elsevier Masson, 2014

[15] L. Deng, F. Janabi-Sharifi, W.J. Wilson, Stability and robustness of visual servoing methods, in: Proceedings of IEEE International Conference on Robotics and Automation, ICRA'02, 2002, pp. 1604-1609.

[16] D. Efimov, A. Polyakov, J.-P. Richard, Interval observer design for estimation and control of time-delay descriptor systems, Eur. J. Control 23 (2015) 26-35.

[17] B. Espiau, F. Chaumette, P. Rives, A new approach to visual servoing in robotics, Geom. Reason. Percept. Action (1993) 106-136, doi:10.1007/3-540-57132-9_8.

[18] E. Fridman, Tutorial on Lyapunov-based methods for time-delay systems, Eur. J. Control 20 (2014) 271-283. 2014/11/01/.

[19] N.R. Gans, S.A. Hutchinson, P.I. Corke, Performance tests for visual servo contro systems, with application to partitioned approaches to visual servo control, Int. J. Robot. Res. 22 (2003) 955-981.

[20] R.A.R.C. Gopura, K. Kiguchi, Y. Li, SUEFUL-7: A 7DOF upper-limb exoskeleton robot with muscle-model-oriented EMG-based control, in: Proceedings of International Conference on Intelligent Robots and Systems, IEEE/RSJ, 2009, pp. 1126-1131.

[21] J. Han, From PID to active disturbance rejection control, IEEE Trans. Ind. Electron. 56 (2009) 900-906.

[22] M. Hashemi, J. Askari, J. Ghaisari, Adaptive control of uncertain nonlinear time delay systems in the presence of actuator failures and applications to chemica reactor systems, Eur. J. Control 29 (2016) 62-73. 2016/05/01/.

[23] A.-C. Huang, M.-C. Chien, Adaptive control of robot manipulators: a unified regressor-free approach, World Scientific, 2010.

[24] S. Hutchinson, G.D. Hager, P.I. Corke, A tutorial on visual servo control, Int. J. Robot. Res. 12 (1996) 651-670.

[25] M. Jin, J. Lee, K.K. Ahn, Continuous nonsingular terminal sliding-mode control of shape memory alloy actuators using time delay estimation, IEEE/ASME Trans. Mechatron. 20 (2015) 899-909.

[26] U. Keller, S. Schölch, U. Albisser, C. Rudhe, A. Curt, R. Riener, et al., Robot-assisted arm assessments in spinal cord injured patients: a consideration of concept study, PloS ONE 10 (2015) e0126948.

[27] H.K. Khalil, Noninear systems, 2, Prentice-Hall, New Jersey, 1996 pp. 5-1.

[28] J. Kim, H. Joe, S.-c. Yu, J.S. Lee, M. Kim, Time-delay controller design for position control of autonomous underwater vehicle under disturbances, IEEE Trans. Ind. Electron. 63 (2016) 1052-1061.

[29] F.L. Lewis, D.M. Dawson, C.T. Abdallah, Robot Manipulator Control: Theory and Practice, CRC Press, 2003.

[30] C. Liu, C.C. Cheah, J.-J.E. Slotine, Adaptive Jacobian tracking control of rigid-link electrically driven robots based on visual task-space information, Automatica 42 (2006) 1491-1501.

31] C.O. Luna, M.H. Rahman, M. Saad, P. Archambault, W.-H. Zhu, Virtual decomposition control of an exoskeleton robot arm, Robotica 34 (2016) 1587-1609.

[32] E. Lundström, A. Terént, J. Borg, Prevalence of disabling spasticity 1 year after first-ever stroke, Eur. J. Neurol. 15 (2008) 533-539. 
[33] E. Malis, F. Chaumette, Theoretical improvements in the stability analysis of a new class of model-free visual servoing methods, IEEE Trans. Robotics Autom. 18 (2002) 176-186.

[34] D.S. Nichols-Larsen, P. Clark, A. Zeringue, A. Greenspan, S. Blanton, Factors influencing stroke survivors' quality of life during subacute recovery, Stroke 36 (2005) 1480-1484.

[35] M.H. Rahman, M.J. Rahman, O. Cristobal, M. Saad, J.-P. Kenné, P.S. Archambault, Development of a whole arm wearable robotic exoskeleton for rehabilitation and to assist upper limb movements, Robotica 33 (2015) 19.

[36] M.A. Rami, M. Schönlein, J. Jordan, Estimation of linear positive systems with unknown time-varying delays, Eur. J. Control 19 (2013) 179-187.

[37] S. Sidney, W.D. Rosamond, V.J. Howard, R.V. Luepker, The "heart disease and stroke statistics-2013 update" and the need for a national cardiovascular surveillance system, Circulation 127 (2013) 21-23.

[38] J.-J.E. Slotine, W. Li, Appl. Nonlinear Control 199 (1991).
[39] H. Tang, Y. Li, Development and active disturbance rejection control of a compliant micro-/nanopositioning piezostage with dual mode, IEEE Trans. Ind. Electron. 61 (2014) 1475-1492.

[40] B. Yao, Adaptive Robust Control of Nonlinear Systems with Application to Control of Mechanical Systems, University of California, Berkeley, 1996.

[41] H. Yazarel, C.-C. Cheah, Task-space adaptive control of robotic manipulators with uncertainties in gravity regressor matrix and kinematics, IEEE Trans. Autom. Control 47 (2002) 1580-1585.

[42] K. Youcef-Toumi, O. Ito, A time delay controller for systems with unknown dynamics, J. Dyn. Syst. Meas. Control 112 (1990) 133-142.

[43] D. Zheng, Z. Ren, J. a. Fang, Stability Analysis of time delayed system with coefficient uncertainty and time delay uncertainty, Eur. J. Control 16 (2010) 5-13. 2010/01/01/. 